\title{
New constituents of Baccharis genistelloides (Lam.) Pers.
}

\author{
Lothar Hennig, ${ }^{\text {a* }}$ Gonzalo Malca Garcia, ${ }^{\text {a }}$ Athanassios Giannis, \\ and Rainer W. Bussmann ${ }^{b}$ \\ ${ }^{a}$ Universität Leipzig, Institut für Organische Chemie, Johannisallee 29, 04103 Leipzig, Germany \\ ${ }^{b}$ William L. Brown Center, Missouri Botanical Garden, P.O. Box 299, \\ St. Louis, MO 63166-0299, USA \\ E-mail: hennig@,chemie.uni-leipzig.de
}

\section{Dedicated to Prof. Heinz Heimgartner on the occasion of his $\mathbf{7 0}^{\text {th }}$ birthday}

\begin{abstract}
A phytochemical investigation of the constituents of the aerial parts of Baccharis genistelloides has resulted in the isolation of the new piquerol derivative $(E)$-4-acetoxy-6-methylene-5-(prop-1en-2-yl)cyclohex-2-enyl 2-methylbut-2-enoate 4, along with two other compounds [foliasalacin $\mathrm{A}_{4}$ 5, and (E)-icosyl 3-(4-hydroxy-3-methoxyphenyl)-acrylate 6], which have not been reported as constituents of this plant until now.
\end{abstract}

Keywords: Baccharis genistelloides, piquerol, foliasalacin $\mathrm{A}_{4}$, icosyl ferulate

\section{Introduction}

Baccharis is a New World genus belonging to the Asteraceae (tribe Astereae). The genus includes more than 400 species, about $90 \%$ of which are located in South America. Many of these species have been used since ancient times as folk remedies for some treatment purposes. Over 100 Baccharis species have been investigated to reveal that they contain many classes of secondary metabolites, e.g. terpenoids, diterpenoids, sesquiterpenes, triterpenoids, essential oils, flavonoids, coumarins, and other phenolic compounds. During the intensive investigation of the chemical components of Baccharis, much attention has been paid to its bioactive constituents. ${ }^{1}$ Baccharis genistelloides (Lam.) Pers. ("Carqueja", "Charara") is used for liver disorders and as an antithermic. Other popular uses include digestive disorders, malaria, diabetes, ulcers, sore throat and tonsillitis, anaemia, diarrhea, indigestion, intestinal worms and leprosy. $B$. genistelloides is also widely used in folk medicine in the form of infusion for its antiinflammatory properties. $^{2-4}$ 
$\beta$-Pinene (15\%), carquejyl acetate (55\%) and carquejol $1(6-7 \%)$ were identified by Naves as the main components of the essential oil of this plant and numerous derivatives have been synthesized to confirm the structure of carquejol. ${ }^{5}$ The relative and absolute configuration of $\mathbf{1}$ was determined by Thomas et al. ${ }^{6}$

In an investigation of the chemical constituents of. B. genistelloides, flavones named eupatrin 2a, cirsimaritin $\mathbf{2 b}$, cirsiliol 2c, hispidulin $\mathbf{2 d}$ and genkwanin $\mathbf{2 e}$, together with the known apigenin $2 \mathbf{f}$ were isolated. ${ }^{7-10}$ It is thought that these flavones are the major active substances in this plant. Quercetin 2g, luteolin $\mathbf{2 h}$, and nepetin $\mathbf{2} \mathbf{i}$ also have been found. ${ }^{11}$ Other flavonoids, e.g. rutin $2 \mathbf{j}$, have been isolated as anti-inflammatory constituents. ${ }^{12}$ Dos Santos et al. ${ }^{13}$ reported the isolation of eupatorine $\mathbf{2 k}$ and the diterpene lactone $\mathbf{3}$ which showed antimicrobial activity. In another study ${ }^{14 a}$ the biological activity of the dilactonic clerodane-diterpene $3^{14 b, c}$ from $B$. genistelloides has also been described.<smiles>C=C(C)[C@H]1C(=C)CC=C[C@H]1O</smiles>

1



$\mathbf{2 a - k}$

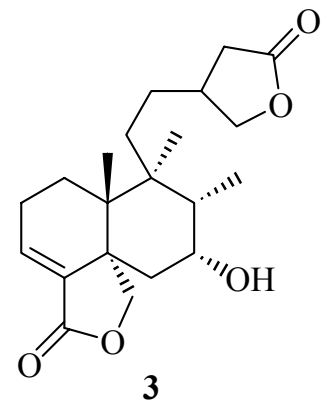

3

\begin{tabular}{ccccccccc}
\hline & & $\mathrm{R}^{1}$ & $\mathrm{R}^{2}$ & $\mathrm{R}^{3}$ & $\mathrm{R}^{4}$ & $\mathrm{R}^{5}$ & $\mathrm{R}^{6}$ & $\mathrm{R}^{7}$ \\
\hline $\mathbf{2 a}$ & Eupatrin & $\mathrm{OH}$ & $\mathrm{OMe}$ & $\mathrm{OMe}$ & $\mathrm{OH}$ & $\mathrm{OMe}$ & $\mathrm{OH}$ & $\mathrm{H}$ \\
$\mathbf{2 b}$ & Cirsimaritin & $\mathrm{OH}$ & $\mathrm{OMe}$ & $\mathrm{OMe}$ & $\mathrm{H}$ & $\mathrm{H}$ & $\mathrm{OH}$ & $\mathrm{H}$ \\
$\mathbf{2 c}$ & Cirsiliol & $\mathrm{OH}$ & $\mathrm{OMe}$ & $\mathrm{OMe}$ & $\mathrm{H}$ & $\mathrm{OH}$ & $\mathrm{OH}$ & $\mathrm{H}$ \\
$\mathbf{2 d}$ & Hispidulin & $\mathrm{H}$ & $\mathrm{OMe}$ & $\mathrm{OH}$ & $\mathrm{H}$ & $\mathrm{H}$ & $\mathrm{OH}$ & $\mathrm{H}$ \\
$\mathbf{2 e}$ & Genkwanin & $\mathrm{OH}$ & $\mathrm{H}$ & $\mathrm{OMe}$ & $\mathrm{H}$ & $\mathrm{H}$ & $\mathrm{OH}$ & $\mathrm{H}$ \\
$\mathbf{2 f}$ & Apigenin & $\mathrm{OH}$ & $\mathrm{H}$ & $\mathrm{OH}$ & $\mathrm{H}$ & $\mathrm{H}$ & $\mathrm{OH}$ & $\mathrm{H}$ \\
$\mathbf{2 g}$ & Quercetin & $\mathrm{OH}$ & $\mathrm{H}$ & $\mathrm{OH}$ & $\mathrm{H}$ & $\mathrm{OH}$ & $\mathrm{OH}$ & $\mathrm{OH}$ \\
$\mathbf{2 h}$ & Luteolin & $\mathrm{OH}$ & $\mathrm{H}$ & $\mathrm{OH}$ & $\mathrm{H}$ & $\mathrm{OH}$ & $\mathrm{OH}$ & $\mathrm{H}$ \\
$\mathbf{2 i}$ & Nepetin & $\mathrm{OH}$ & $\mathrm{OMe}$ & $\mathrm{OH}$ & $\mathrm{H}$ & $\mathrm{OH}$ & $\mathrm{OH}$ & $\mathrm{H}$ \\
$\mathbf{2 j}$ & Rutin & $\mathrm{OH}$ & $\mathrm{H}$ & $\mathrm{OH}$ & $\mathrm{H}$ & $\mathrm{OH}$ & $\mathrm{OH}$ & $\mathrm{H}$ \\
$\mathbf{2 k}$ & Eupatorin & $\mathrm{OH}$ & $\mathrm{OMe}$ & $\mathrm{OMe}$ & $\mathrm{H}$ & $\mathrm{OH}$ & $\mathrm{OMe}$ & $\mathrm{H}$ \\
\hline
\end{tabular}

In the present study, we describe a new piquerol derivative and two other unknown compounds of Baccharis genistelloides. 


\section{Results and Discussion}

From the n-hexane extract of the aerial part of Baccharis genistelloides (collected in Huamachuco, Northern Peru) we have isolated three new constituents of this plant after careful chromatography.

First of all, the n-hexane extract was purified by column chromatography (silicagel; nhexane-acetone 4:1) to give different fractions. A part of it (fractions 22-38) was then purified with n-hexane-ethyl acetate (5:2) followed by n-hexane-acetone $(10: 1)$ to give $(E)$-4-Acetoxy-6methylene-5-(prop-1-en-2-yl)cyclohex-2-enyl 2-methylbut-2-enoate 4 as a colourless oil.
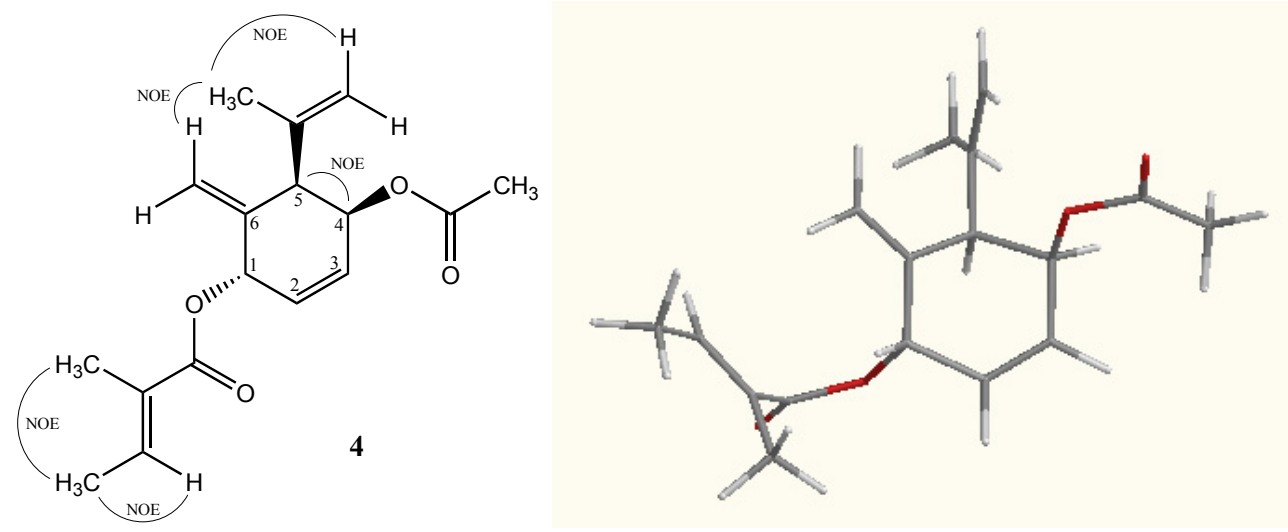

The structure of $\mathbf{4}$ has been identified by a combination of spectroscopic methods. The full NMR assignment was made by ${ }^{1} \mathrm{H},{ }^{13} \mathrm{C}$, APT, gCOSY, gHSQC, gHMBC and NOESY experiments and is given in the experimental section and the supplementary material. In the HMBC experiment long-range correlations between the proton in 5-position (3.38 ppm) and the ring carbon atoms C-4, C-6, C-1, C-3 and all carbons of the side chains in 5- and 6-position were observed. NOE correlations have been found between the ring protons H-4 and H-5 at 5.44 and $3.38 \mathrm{ppm}$, respectively, but not between $\mathrm{H}-1$ and $\mathrm{H}-5$. Other important NOE correlations are shown in the formula. The $E$-configuartion of the substituent in 1-position can be concluded from these NOE's. Therefore, the stereostructure can be described best with the model shown above.

Two similar terpene derivatives already have been found by $\mathrm{F}$. Bohlmann et al. in the roots of Baccharis trimera (Less.) DC. ${ }^{15}$ Both compounds with unusual terpene carbon skeleton are derivatives of piquerol $\mathrm{A}$, and one of them was also identified as a constituent in the roots of Piqueria trinervia Cav. ${ }^{16}$ Piquerol A represents a hydroxy substituted carquejol with high biological potential. ${ }^{17}$ Its structure was determined with different spectroscopic methods ${ }^{18}$ and Xray analysis. ${ }^{19}$ Piquerol $\mathrm{A}$ and carquejol as well are probably formed starting from $\beta$-pinene (Scheme 1). 


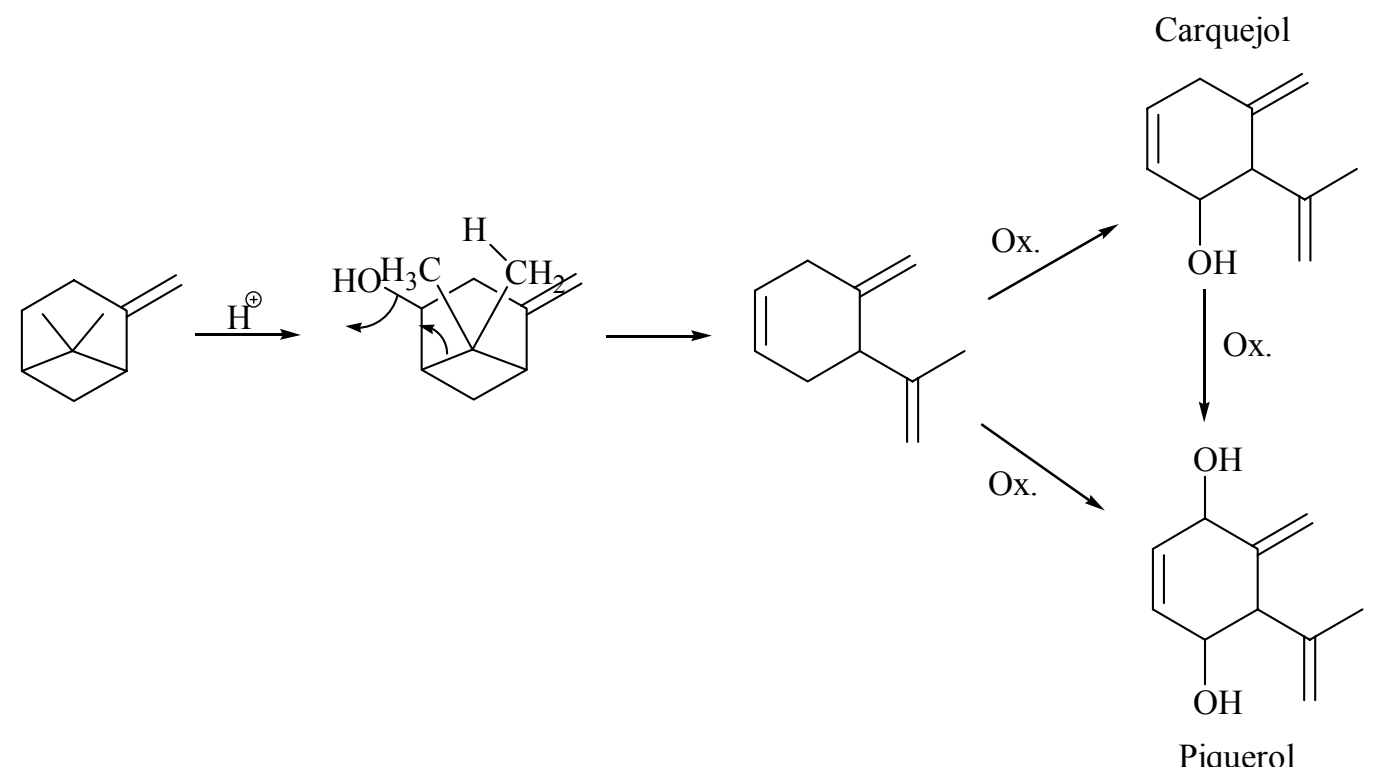

\section{Scheme 1}

Furthermore, we isolated from the aerial part of Baccharis genistelloides Foliasalacyin $\mathrm{A}_{4} \mathbf{5}$ as a new constituent of this plant. The triterpene Foliasalacyin $\mathrm{A}_{4} \mathbf{5}$ was obtained as a white powder after column chromatography with n-hexane-ethyl acetate $(5: 3)$ followed by n-hexaneethyl acetate (5:4). Other triterpenoid compounds (phytol, $\beta$-sitosterol and stigmasterol) already have been identified during the investigation of B. serrifolia DC. and B. genistelloides. ${ }^{20,21}$ The combination of different NMR spectra of 5 showed eight methyl groups, an olefinic methylene group, a methine carbon bearing a hydroxy group, and a quaternary carbon which is also connected to a hydroxy group (Supplementary Material). The final structure could be proved by comparison with literature results.

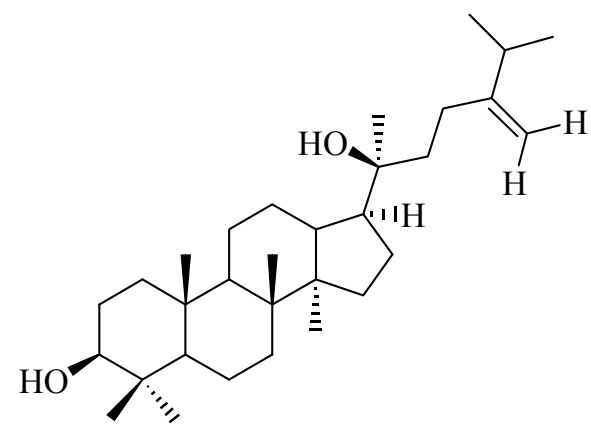

5

Foliasalacin $\mathrm{A}_{4}$ is a dammarane-type triterpene which recently was isolated for the first time from the leaves of Solacia chinensis L. collected in Thailand. ${ }^{22}$ All our recorded spectra of 5 are 
in total agreement with the spectra and characterization of the stereostructure described in literature..$^{22}$

Finally, purification of fractions $95-108$ of the n-hexane-acetone 4:1 eluate furnished the icosyl ferulate $\mathbf{6}$ (column chromatography with n-hexane-acetone 2:1) as a slightly yellow solid. The structure of $\mathbf{6}$ was proved by NMR, IR, UV-Vis and HRMS. The elemental constitution could be calculated from the molecular ion and is in accordance with the NMR spectra. A coupling constant of $16.0 \mathrm{~Hz}$ of the olefinic protons in the proton spectrum showed (E)configuration. The position of hydroxy and methoxy group in the aromatic ring system was identified by long-range correlations in the HMBC experiment.

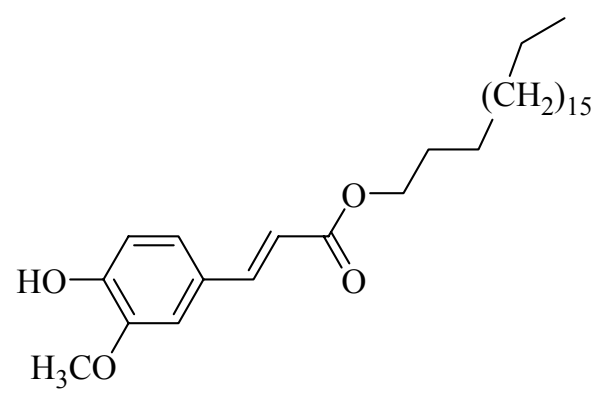

6

The antioxidant ferulic acid derivatives are ubiquitous in plants and abundant in fruits and vegetables. For example, different ferulates, including the icosyl ferulate 6, were isolated from cork layers of Solanum tuberosum L. and Pseudotsuga menziesii (Mirb.) Franco. ${ }^{23}$ Ferulic acid esters have been tested for tumor cell proliferation, cyclooxygenase enzymes (COX-1 and COX2) and lipid peroxidation inhibitory activities in vitro. In the tumor cell proliferation assay, some of these esters showed excellent growth inhibition of colon cancer cells. In COX enzyme assays, ferulic acid esters significantly inhibited both COX-1 and COX-2 enzymes. ${ }^{24}$

\section{Experimental Section}

General. NMR spectra were recorded with a Varian Mercury Plus 400 spectrometer. Residual solvent signals were used as internal chemical shift references for proton (DMSO- $d_{6}: \delta 2.50$ ppm; $\mathrm{CDCl}_{3}: \delta 7.26 \mathrm{ppm}$ ) and carbon (DMSO- $d_{6}: \delta 39.52 \mathrm{ppm} ; \mathrm{CDCl}_{3}: \delta 77.16 \mathrm{ppm}$ ). The full assignment is attached in the supplementary information. Mass spectra were measured on a VG ZAB-HSQ (VG Analytics) and HRMS spectra in positive mode on a FT-ICR-MS APEX II (Bruker Daltonics) spectrometer. IR spectra were recorded on a spectrophotometer Genesis FTIR Unicam Analytical System (ATI Mattson) with $\mathrm{KBr}$ pellets. Elemental analyses were performed on a Heraeus CHNO Rapid Analyzer. UV-Vis spectra were recorded on a Beckman DU 650; $\lambda_{\max }$ in $\mathrm{nm}(\log \varepsilon)$. All separations were carried out in carefully purified and dried solvents and were monitored by thin-layer chromatography (TLC) on plates of Silufol UV/VIS $254 \mathrm{~nm}$. 
Preparative column chromatography was carried out on neutral silica gel (MERCK 70-230 mesh) in gradient regime.

\section{Plant material}

The aerial part of B. genistelloides (Lam.) Pers. was collected in March 2009 at Huamachuco (José Faustino Sánchez Carrión Province - Perú) and identified by Botanist Eric RodriguezRodriguez [National University of Trujillo; Herbarium Truxillense (HUT)]. A voucher specimen No. 50002 (HUT) has been deposited at the Herbarium Truxillense (HUT) of the National University of Trujillo (Perú).

\section{Isolation}

The air-dried aerial parts of the plant (103.52 g) were extracted with n-hexane $(5 \mathrm{~L})$ by cold maceration for 5 days. The resulting extract was concentrated in vacuo to afford a residue of 2.61 g. This residue was stored at $-10{ }^{\circ} \mathrm{C}$ until use for column chromatography (CC).

The $\mathrm{n}$-hexane extract $(2.61 \mathrm{~g})$ was purified by $\mathrm{CC}$ on silcagel with $\mathrm{n}$-hexane-acetone (4:1) according to the TLC profile resulting in 115 fractions. Fractions 22-38 were eluted by repeated $\mathrm{CC}$ with n-hexane-ethyl acetate $(5: 2)$ to give six new fractions. Out of these the second fraction gave $33.8 \mathrm{mg}$ of 4 (Rf. 0.48) using $\mathrm{CC}$ with n-hexane-acetone (10:1). The sixth fraction was purified with n-hexane-ethyl acetate $(5: 3)$ to give four other fractions. Out of these fractions the first one was eluted with n-hexane-ethyl acetate (5:4) to give $21.5 \mathrm{mg}$ of 5 (Rf. 0.44). Fractions 95-108 from the original n-hexane-acetone 4:1 eluate were purified by CC with n-hexaneacetone (2:1) to give $40.0 \mathrm{mg}$ of 6 (Rf. 0.5 ).

(E)-4-Acetoxy-6-methylene-5-(prop-1-en-2-yl)cyclohex-2-enyl 2-methylbut-2-enoate (4). Colourless oil; $\mathrm{C}_{17} \mathrm{H}_{22} \mathrm{O}_{4}$ (290.35). ${ }^{1} \mathrm{H}$ NMR $\left(\mathrm{CDCl}_{3}\right) \delta 1.73$ (s (br), $\left.3 \mathrm{H}, \mathrm{CH}_{3}\right), 1.79$ (d, $J=6.9$ $\mathrm{Hz}, 3 \mathrm{H}, \mathrm{CH}_{3}$ ), 1.82 (s (br), $3 \mathrm{H}, \mathrm{CH}_{3}$ ), 2.05 (s, $3 \mathrm{H}, \mathrm{CH}_{3} \mathrm{COO}$ ), 3.38 (d, J=5.2 Hz, $1 \mathrm{H}, \mathrm{H}-5$ ), 4.92 (s (br), $1 \mathrm{H},=\mathrm{CH}_{2}$ ), 4.97 (s (br), $\left.1 \mathrm{H},=\mathrm{CH}_{2}\right), 5.44$ (m, $\left.1 \mathrm{H}, \mathrm{H}-4\right), 5.21\left(\mathrm{~s}, 1 \mathrm{H},=\mathrm{CH}_{2}\right), 5.32$ $\left(\mathrm{s}, 1 \mathrm{H},=\mathrm{CH}_{2}\right), 5.82(\mathrm{~m}, 1 \mathrm{H}, \mathrm{H}-2), 5.83(1 \mathrm{H}, \mathrm{H}-1), 6.00(\mathrm{~m}, 1 \mathrm{H}, \mathrm{H}-3), 6.86(\mathrm{q}, J=6.9 \mathrm{~Hz}, 1 \mathrm{H}$, $=\mathrm{CH}-) .{ }^{13} \mathrm{C} \mathrm{NMR}\left(\mathrm{CDCl}_{3}\right) \delta 12.11\left(\mathrm{CH}_{3}\right), 14.57\left(\mathrm{CH}_{3}\right), 21.29\left(\mathrm{CH}_{3} \mathrm{COO}\right), 23.24\left(\mathrm{CH}_{3}\right), 50.10(\mathrm{C}-$ 5), 69.38 (C-1), $70.95(\mathrm{C}-4), 114.61\left(=\mathrm{CH}_{2}\right), 116.84\left(=\mathrm{CH}_{2}\right), 128.52(\mathrm{C}-2), 128.71(=\mathrm{Cq}), 130.64$ (C-3), 137.97 (=CH-), 141.07 (=Cq), 141.34 (C-6), 167.45 (COO), 170.58 (COO); IR $v_{\max } 1738$, $1709,1650 \mathrm{~cm}^{-1}$; HRMS $m / z 313.14108\left(\mathrm{M}^{+}+\mathrm{Na}\right)$.

$(3 S, 8 R, 10 R, 14 R, 17 S)-17-((S)-2-h y d r o x y-6-m e t h y l-5-m e t h y l e n e h e p t a n-2-y l)-4,4,8,10,14-$ pentamethyl-hexadecahydro-1H-cyclopenta[a]phenanthren-3-ol (Foliasalacin $\left.\mathbf{A}_{4}\right)$ (5). White powder; $\mathrm{C}_{31} \mathrm{H}_{54} \mathrm{O}_{2}$ (458.76). ${ }^{1} \mathrm{H}$ NMR $\left(\mathrm{CDCl}_{3}\right) \delta 0.73(1 \mathrm{H} ; \mathrm{H}-5), 0.77\left(\mathrm{~s}, 3 \mathrm{H}, \mathrm{CH}_{3}-28\right)$; 0.85 (s, $\left.3 \mathrm{H}, \mathrm{CH}_{3}-19\right), 0.88$ (s, $\left.3 \mathrm{H}, \mathrm{CH}_{3}-18\right), 0.96$ (s, $\left.3 \mathrm{H}, \mathrm{CH}_{3}-30\right), 0.96 / 1.70$ (m, $2 \mathrm{H}, \mathrm{CH}_{2}-1$ ), 0.97 (s, $\left.3 \mathrm{H}, \mathrm{CH}_{3}-29\right), 1.04$ (d, $\left.J=6.8 \mathrm{~Hz}, 6 \mathrm{H}, \mathrm{CH}_{3}-26,27\right), 1.08 / 1.48$ (m, $\left.2 \mathrm{H}, \mathrm{CH}_{2}-15\right), 1.16$ (s, $3 \mathrm{H}, \mathrm{CH}_{3}-21$ ), 1.25/1.51 (m, $\left.2 \mathrm{H}, \mathrm{CH}_{2}-11\right), 1.28 / 1.53$ (m, 2H, $\mathrm{CH}_{2}-7$ ), 1.32 (m, $1 \mathrm{H}, \mathrm{CH}-9$ ), 1.35/1.81 (m, $\left.2 \mathrm{H}, \mathrm{CH}_{2}-16\right), 1.44 / 1.52\left(\mathrm{~m}, 2 \mathrm{H}, \mathrm{CH}_{2}-6\right), 1.51 / 1.76$ (m, $\left.2 \mathrm{H}, \mathrm{CH}_{2}-12\right)$, 1.58/1.63 (m, $\left.2 \mathrm{H}, \mathrm{CH}_{2}-2\right), 1.59 / 1.61\left(\mathrm{~m}, 2 \mathrm{H}, \mathrm{CH}_{2}-22\right), 1.65$ (m, $\left.1 \mathrm{H}, \mathrm{CH}-13\right), 1.76$ (m, $\left.1 \mathrm{H}, \mathrm{CH}-17\right)$, 
2.08/2.10 (m, $2 \mathrm{H}, \mathrm{CH}_{2}-23$ ), 2.26 (sept, $\left.J=6.8 \mathrm{~Hz}, 1 \mathrm{H}, \mathrm{CH}-25\right), 3.20$ (dd, $J=11.2 \mathrm{~Hz}, J=5.2$ $\mathrm{Hz}, 1 \mathrm{H}, \mathrm{CH}-3$ ), 4.69 (s (br), $\left.1 \mathrm{H},=\mathrm{CH}_{2}\right), 4.75$ (s (br), $\left.1 \mathrm{H},=\mathrm{CH}_{2}\right) .{ }^{13} \mathrm{C} \mathrm{NMR}\left(\mathrm{CDCl}_{3}\right) \delta 15.51$ $\left(\mathrm{CH}_{3}-28\right), 15.62\left(\mathrm{CH}_{3}-30\right), 16.37\left(\mathrm{CH}_{3}-19\right), 16.64\left(\mathrm{CH}_{3}-18\right), 18.44\left(\mathrm{CH}_{2}-6\right), 21.71\left(\mathrm{CH}_{2}-11\right)$, $22.08\left(\mathrm{CH}_{3}-27\right), 22.11\left(\mathrm{CH}_{3}-26\right), 24.94\left(\mathrm{CH}_{2}-12\right), 25.53\left(\mathrm{CH}_{3}-21\right), 27.57\left(\mathrm{CH}_{2}-2\right), 27.69\left(\mathrm{CH}_{2}-\right.$ 16), $28.15\left(\mathrm{CH}_{3}-29\right), 28.54\left(\mathrm{CH}_{2}-23\right), 31.37\left(\mathrm{CH}_{2}-15\right), 34.18(\mathrm{CH}-25), 35.38\left(\mathrm{CH}_{2}-7\right), 37.29(\mathrm{C}-$ 10), 39.13 (C-4), $39.20\left(\mathrm{CH}_{2}-1\right), 39.56\left(\mathrm{CH}_{2}-22\right), 40.53$ (C-8), $42.51(\mathrm{CH}-13), 49.93(\mathrm{CH}-17)$, 50.50 (C-14), 50.79 (CH-9), 56.02 (CH-5), 75.49 (C-20), 79.12 (CH-3), $106.31\left(\mathrm{CH}_{2}-31\right), 156.69$ (C-24); IR $v_{\max } 3434,2955,2869,1642 \mathrm{~cm}^{-1}$.

(E)-Icosyl 3-(4-hydroxy-3-methoxyphenyl)-acrylate (6). Slightly yellow solid; $\mathrm{C}_{30} \mathrm{H}_{50} \mathrm{O}_{4}$ (474.72). ${ }^{1} \mathrm{H} \mathrm{NMR}\left(\mathrm{CDCl}_{3}\right) \delta 0.86\left(\mathrm{t}, J=6.8 \mathrm{~Hz}, 3 \mathrm{H}, \mathrm{CH}_{3}\right), 1.22\left(\mathrm{~m}, 30 \mathrm{H}, 15 \mathrm{xCH}_{2}\right), 1.29(\mathrm{~m}, 2$ $\left.\mathrm{H}, \mathrm{CH}_{2}\right), 1.39\left(\mathrm{~m}, 2 \mathrm{H}, \mathrm{CH}_{2}\right), 1.70\left(\mathrm{~m}, 2 \mathrm{H}, \mathrm{CH}_{2}\right), 3.94\left(\mathrm{~s}, 3 \mathrm{H}, \mathrm{OCH}_{3}\right), 4.19(\mathrm{t}, J=6.8 \mathrm{~Hz}, 2 \mathrm{H}$, $\mathrm{OCH}_{2}$ ), 5.84 (s (br), $\left.1 \mathrm{H}, \mathrm{OH}\right), 6.29$ (d, $\left.J=16.0 \mathrm{~Hz}, 1 \mathrm{H},=\mathrm{CH}-\right), 6.91$ (d, $\left.J=8.4 \mathrm{~Hz}, 1 \mathrm{H}, \mathrm{Ph}\right)$, $7.03(\mathrm{~d}, J=2.0 \mathrm{~Hz}, 1 \mathrm{H}, \mathrm{Ph}), 7.07(\mathrm{dd}, J=8.4 \mathrm{~Hz}, \mathrm{~J}=2.0 \mathrm{~Hz}, 1 \mathrm{H}, \mathrm{Ph}), 7.61(\mathrm{~d}, J=16.0 \mathrm{~Hz}, 1$ $\mathrm{H},=\mathrm{CH}-) .{ }^{13} \mathrm{C} \mathrm{NMR}\left(\mathrm{CDCl}_{3}\right) \delta 14.27\left(\mathrm{CH}_{3}\right), 22.84\left(\mathrm{CH}_{2}\right), 26.15\left(\mathrm{CH}_{2}\right), 28.94\left(\mathrm{CH}_{2}\right), 29.46$ $\left(\mathrm{CH}_{2}\right), 29.52\left(\mathrm{CH}_{2}\right), 29.70\left(\mathrm{CH}_{2}\right), 29.75\left(\mathrm{CH}_{2}\right), 29.81\left(2 \mathrm{xCH}_{2}\right), 29.85\left(9 \mathrm{xCH}_{2}\right), 56.09\left(\mathrm{OCH}_{3}\right)$, $64.77\left(\mathrm{OCH}_{2}\right), 109.44$ (C-2-Ph), 114.84 (C-5-Ph), 115.87 (=CH-), 123.19 (C-6-Ph), 127.23 (C-1$\mathrm{Ph}), 144.77$ (=CH-), 146.90 (C-3-Ph), 148.04 (C-4-Ph), 167.54 (CO); HRMS m/z 497.36180 (M $+\mathrm{Na}), 971.73813\left(2 \mathrm{M}^{+}+\mathrm{Na}\right)$; IR $v_{\max } 3427,2917,2849,1713 \mathrm{~cm}^{-1} ; \mathrm{UV}-\mathrm{Vis}\left(\mathrm{CHCl}_{3}\right) \lambda=320$ (4.37), 295 (4.10), 243 (4.00).

\section{Acknowledgements}

We are grateful to Deutscher Akademischer Austauschdienst (DAAD) for financial support (G. Malca Garcia).

\section{References}

1. Martinez, M. J. A.; Bessa, A. L.; Benito, P. B. In Studies in Natural Products Chemistry; Vol. 30, Elsevier: Amsterdam, Boston, Heidelberg, London, New York, Oxford, Paris, San Diego, San Francisco, Singapore, Sydney, Tokyo, 2005, pp 703-759.

2. (a) Simoes, C. M. O.; Mentz, L. A.; Schenkel, E. P.; Irgang, B. E.; Stehmann, J. R. Plantas da Medicina Popular no Rio Grande do Sul, Ed. Da Universidade: Porto Alegre, 1986. (b) Ruiz, A.L.T.G.; Taffarello, D.; Souza, V. H. S.; Carvalho, J. E. Revista Brasileira de Farmacognosia 2008, 18, 295. (c) Coelho, M. G. P.; Reis, P. A.; Gava, V. B.; Marques, P. R.; Gayer, C. R.; Laranja, G. A. T.; Felzenswalb, I.; Sabino, K.C.C. Toxicology Lett. 2004, 154, 69.

3. (a) Sousa, M. P.; Matos, N. E. O.; Matos, M. F. J. A.; Machado, M. I. L.; Craveiro, A. A. Constituintes Quimicos Activos de Plantas Medicinais Brasileiras, Fortaleza Edicoes UFC: Brasil, 1991. (b) Gonzales, E.; Iglesias, I.; Carretero, E.; Villar, A. J. of Ethnopharmacology 2000, 70, 329. (c) Abad, M.J.; Bermejo, P.; Gonzales, E.; Iglesias, I.; Irurzun, A.; Carrasco, L. Gen. Pharm. 1999, 32, 499. 
4. (a) Bussmann, R. W.; Sharon, D. Plants of the four winds - the magic and medicinal flora of Peru, Editorial GRAFICART srl, Trujillo-Peru, 2007, p 120. (b) Marques, V.; Farah, A. Food Chem. 2009, 113, 1370.

5. (a) Naves, Y. R. Compt. rend. 1959, 249, 562. (b) Naves, Y. R. Bull. Soc. Chim. France, 1959, 1871. (c) Naves, Y. R. France et Ses Parfums 1960, 3(15), 30. (d) Naves, Y. R.; Grampoloff, A. V. Bull. Soc. Chim. France 1961, 1921. (e) Feretti-Alloise, M. G.; JacotGuillarmod, A.; Naves, Y. R. Helv. Chim. Acta 1970, 53(2), 201. (f) Naves, Y. R. Helv. Chim. Acta 1962, 45, 1598.

6. (a) Thomas, A. F. Helv. Chim. Acta 1967, 50, 963. (b) Snatzke, G.; Thomas, A. F.; Ohloff, G. Helv. Chim. Acta 1969, 52, 1253.

7. Herz, W.; Pilotti, A. M.; Söderholm, A. C.; Shuhama, I. K.; Vichnewski, W. J. Org. Chem. 1977, 42, 3913.

8. Kuroyanagi, M.; Fujita, K.; Kazaoka, M.; Matsumoto, S.; Veno, A.; Fukushima, S.; Katsuoka, M. Chem. Pharm. Bull. 1985, 33, 5075.

9. Rendon, W.; Vila, J. L. Rev. Boliv. Quim. 1995, 12, 13.

10. Nakasugi, T.; Komai, K. J. Agric. Food Chem. 1998, 46, 2560.

11. Soike, H.; Leng-Peschlow, E. Planta Med. 1987, 53, 37.

12. Gene, R. M.; Castaño, C.; Adzet, T.; Marin, E.; Parella, T.; Cañigeral, S. Planta Med. 1996, $62,232$.

13. Dos Santos, D.; Sarli, S. J.; Vichnewski, W.; Bulhoes, M. S.; De Freitas, H. Rev. Fac. Farm. Odontol. Ribeirao Preto 1980, 17, 43.

14. (a) Brandao, L. M.; Gamberini, M. T.; Roque, N. F.; Lima-Landman, M. T.; Souccar, C.; Lapa, A. J. Phytochemistry 2000, 55, 617; (b) Bohlmann, F.; Knauf, W.; King, R.M.; Robinson, H. Phytochemistry 1979, 18, 1011. (c) Suttisri, R.; Kinghorn, A. D.; Wright, A. D., Sticher, O. Phytochemistry 1994, 35, 443.

15. Bohlmann, F.; Zdero, C. Tetrahedron Lett. 1969, 2419.

16. Bohlmann, F.; Suwita, A. Phytochem. 1978, 17, 560.

17. Mendoza, J. L.; Jimenez, M.; Lotina-Hennsen, B. Pestic. Sci. 1994, 40, 37 and literature cited therein.

18. Romo, J.; Romo de Vivar, A.; Quijano, L.; Rios, T.; Diaz, E. Rev. Latinamericano de Quimica 1970, 1, 72.

19. Soriano Garcia, M.; Jimenez, M.; Gonzalez de la Parra, M.; Hernandez, A.; Schatz, M.; Capana, C. Chem. Lett. 1983, 617.

20. Daily, A.; Wagner, H.; Seligmann, O. Fitoterapia 1984, 55, 236.

21. El Dahmy, I. Sci. Pharm. 1994, 62, 67.

22. Yoshikawa, M.; Zhang, Y.; Wang, T.; Nakamura, S.; Matsuda, H. Chem. Pharm. Bull. 2008, $56,915$.

23. Adamovics, J. A.; Johnson, G.; Stermitz, F. R. Phytochem. 1977, 16(7), 1089.

24. Jayaprakasam, B.; Vanisree, M.; Zhang, Y.; Dewitt, D. L.; Nair, M. G. J. Agric. Food Chem. 2006, 54(15), 5375. 